\title{
A case with Rubinstein-Taybi syndrome: A novel frameshift mutation in the CREBBP gene
}

\author{
Metin Eser ${ }^{1}$, Akif Ayaz², Gözde Yeşil ${ }^{3}$ \\ ${ }^{1}$ Department of Medical Genetics, Aydin State Hospital, Aydin; ${ }^{2}$ Department of Medical Genetics, Adana Numune Training \\ and Research Hospital, Adana; ${ }^{3}$ Department of Genetics, Bezmialem Vakif University, School of Medicine, Istanbul, Turkey \\ E-mail: meserdr@hotmail.com \\ Received: 10th February 2017, Revised: 22th March 2017, Accepted: 19th May 2017
}

SUMMARY: Eser M, Ayaz A, Yeşil G. A case with Rubinstein-Taybi syndrome: A novel frameshift mutation in the CREBBP gene. Turk J Pediatr 2017; 59: 601-603.

Rubinstein-Taybi syndrome (RSTS) is a developmental disorder characterized by a wide spectrum of multiple congenital anomalies and cognitive impairment. RSTS is primarily due to mutations in CREBBP (approximately $55 \%$ of cases) or EP300 (approximately $8 \%$ of cases) genes. A 2 month-old boy had atypical facial findings such as low anterior hairline, triangular face, hirsutism on forehead, down-slanting palpebral fissures, beaked nose, broad nasal bridge, triangular mouth and pointed chin and skeletal finding including broad great thumbs and halluces, and accessory nipple. With this paper, we reported a novel frameshift mutation which is led to premature stop codon in CREBBP gene. As a result, c.2057dupC, reported in this paper enlarges the molecular spectrum of disease-causing CREBBP gene.

Key words: Rubinstein-Taybi syndrome, CREBBP, c.2057dupC.

Rubinstein-Taybi Syndrome (RSTS; \#180849; \#613684) is a rare $(1: 100,000-125,000)$ autosomal-dominant congenital disorder characterized by facial dysmorphic features such as prominent beaked nose, columella below the alae nasi and down-slanting palpebral fissures. The cardinal findings of RSTS are skeletal abnormalities (broad thumbs and halluces), postnatal growth retardation and intellectual disability (ranging from mild to severe).1,2 Besides congenital heart defects, genitourinary and central nervous system (CNS) malformations, skin anomalies and increased predisposition to cancer (especially tumors of neural crest origin), are among the other manifestations of RSTS..$^{1,3}$

Inheritance pattern of RSTS is autosomal dominant with variable expression, but reports of transmission are rare, almost all cases are de novo ${ }^{4}$. The genetic etiology of RSTS is heterogeneous and partially known. In approximately $55 \%$ of RSTS cases, mutations in CAMP response element-binding protein (CREBBP), located on chromosome 16p13.3, are reported ${ }^{1}$. The second gene described in RSTS is E1A-associated protein p300 (EP300, encoding p300), located on chromosome 22q13.2. Mutations in EP300 have been identified in up to $8 \%$ of the cases with RSTS. ${ }^{5,6}$ In about $40 \%$ of clinically diagnosed cases with RTS, the genetic cause remains unknown.

Herein we report a RTS patient with a novel mutation of the CREBBP gene (c.2057dupC) who presented with atypical facial appearance, feeding difficulties and recurrent respiratory infection.

\section{Case Report}

A 2 month-old boy was referred to our department due to his atypical facial appearance and feeding difficulties. His mother was 39 -years old and had hypothyroidism. There was no relevant family history. He was born at 39 weeks from a twin pregnancy with a birth weight of $3000 \mathrm{~g}$ (25-50th percentile). The second fetus deceased at 20 weeks gestational age. Because of respiratory distress and feeding difficulties, he stayed at the intensive care unit for approximately one month. $\mathrm{He}$ experienced recurrent respiratory infection and seizures. At 2 months of age, his height was $54 \mathrm{~cm}$ (3-10p), weight 3,500 g (3p) and 
head circumference $33 \mathrm{~cm}(<3 p)$. Besides microcephaly, he had large anterior fontanelle, low anterior hairline, triangular face, hirsutism on forehead, downslanting palpebral fissures, beaked nose, broad nasal bridge, triangular mouth, pointed chin, broad great thumbs and halluces, deep plantar creases between 1st and 2nd toes, capillary hemangioma on back of neck and accessory nipple below right nipple (Fig. 1A-C). Other observed findings included gastroesophageal reflux on esophagography and minimal secundum ASD.

Some clinical findings of the patient such as microcephaly, beaked nose, broad great thumbs/halluces and short stature overlap with RSTS. Sequencing of CREBBP was performed by next-generation sequencing technique. The sequencing reactions were performed using the MiSeq Illumina sequencer (Illumina, San Diego, CA). Data analysis was performed by MiSeq Reporter. Sequencing of CREBBP revealed a C duplication at c.2057 position in the CREBBP gene (c.2057dupC), a frameshift mutation predicted to result in premature termination at the 726 th amino acid of CREB binding protein
(p.Ala687SerfsTer*39). A written informed consent for the publication of the patient's pictures was given by his parents.

\section{Discussion}

RSTS was described in 1963 by Rubinstein and Taybi $^{4}$ and found to be caused first by mutations in $C R E B B P^{7}$. CREB-binding protein $(\mathrm{CBP})$ is a large protein which includes a bromodomain conserved structural unit critical for protein-protein interactions. The protein serves as a transcriptional coactivator associated with cell development and growth. Chrivia et al. ${ }^{8}$ reported the discovery of a nuclear transcriptional coactivator protein, CBP, that binds specifically to the PKA-phosphorylated form of the CREB protein. But CBP also interacts with a large number of other proteins. By means of CBP, it forms a bridge between the DNA-binding transcription factors and the RNA polymerase II complex. In addition, with intrinsic histone acetyl transferase (HAT) activity, CBP opens the chromatin structure of related locus. P300 protein which is encoded by second minor gene, EP300, identified
A

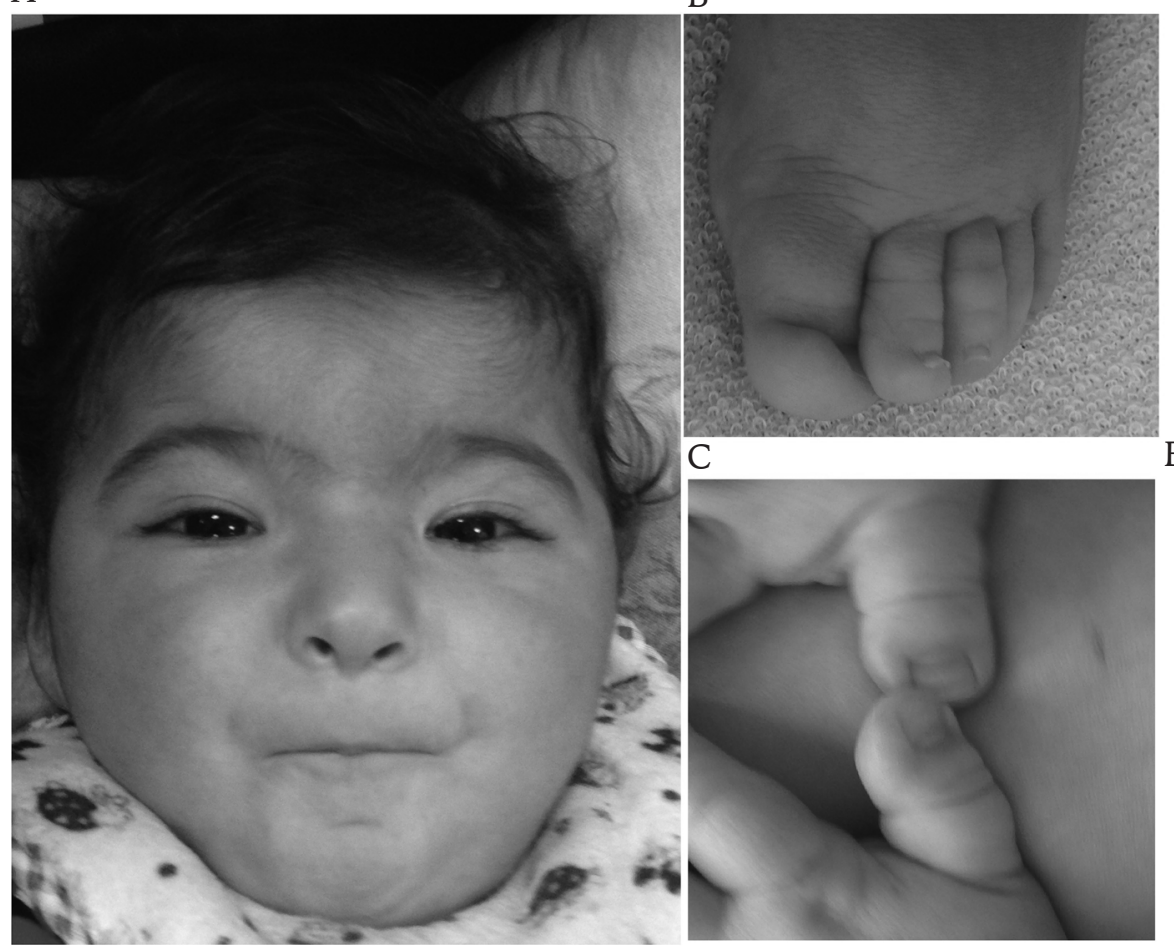

$\mathrm{D}$

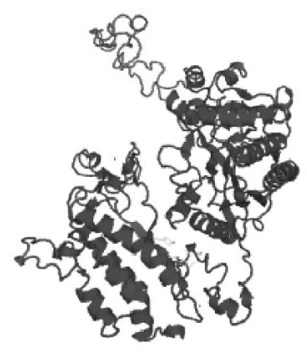

E

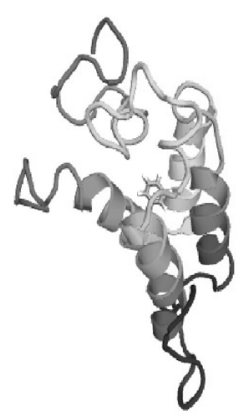

Fig. 1. (A) Facial view from the front. The patient had low anterior hairline, triangular face, hirsutism on forehead, down slanting palpebral fissures, beaked nose, broad nasal bridge, triangular mouth, pointed chin. (B) broad great thumbs and (C) halluces. (D) Molecular modelling of CREB-binding protein. (E) Molecular modelling of mutant, p.Ala687SerfsTer*39, CREB-binding protein 
in RSTS shares homology with CBP. Both proteins have HAT domain and are functional partners in the co-activation of the transcription process. Through histone acetylation, they modify chromatin structure and regulate the expression of a large number of genes. Despite high homology between CBP and P300, both proteins have distinct cellular functions and cannot always replace one another. ${ }^{9,10}$

As far as our knowledge, the mutation presented here, c.2057dupC (p.Ala687SerfsTer*39), has not been reported in the literature to date. Although it was not possible to determine if the mutation was de novo owing to unavailable parental DNA, this mutation is likely to be pathogenic because it leads to premature stop codon. The mutant protein predicted to be free from bromodomain which is important for protein-protein interactions and HAT domain which is a critical fragment for expression of a variety of genes. In addition, these mutant transcripts are targeted for rapid degradation by nonsense-mediated decay (NMD). Molecular modeling of both CREB-binding protein and mutant, p.Ala687SerfsTer*39 CREB-binding protein are shown in Figure $1 \mathrm{D}$ and Figure $1 \mathrm{E}$, respectively.

As for CREBBP gene mutation variety, from chromosomal rearrangements to point mutations located nearly the entire length of the gene are reported. To date, $>300$ mutations are reported in CREBBP according to HGMD (November 2016). ${ }^{11}$ The mutation spectrum is represented mostly from nonsense and missense (103), and splice site (24) heterozygous mutations. Small deletions or small insertions (96) and gross deletions or gross insertions (89) also have been reported.

There are some phenotypic differences between patients with CREBBP and EP300 mutations, particularly the skeletal findings and intellectual development. ${ }^{12}$ In patients with EP300 mutation, skeletal findings and intellectual development also appears to be less affected. In our case, short stature, broad great thumbs and halluces were prominently present; more compatible with CREBBP mutation. In addition, when he was 2 year-old, psychometric evaluation was reported as abnormal but findings were not detailed.

As a result, we present a case with RSTS, which is rare, along with clinical findings and a novel frameshift mutation predicted to result in premature termination, c.2057dupC, in CREBBP gene. In addition, it is important that the mutation reported here enlarges the molecular spectrum of disease-causing $C R E B B P$ gene. To enlarge this spectrum and detail genotype-phenotype correlation, clinically diagnosed cases with RSTS should be screened for CREBBP and EP300 gene mutations.

\section{Acknowledgements}

We would like to thanks the patient's parents for their participation.

\section{REFERENCES}

1. Hennekam RC. Rubinstein-Taybi syndrome. Eur J Hum Genet 2006; 14: 981-985.

2. Stevens CA, Hennekam RC, Blackburn BL. Growth in the Rubinstein-Taybi syndrome. Am J Med Genet Suppl 1990; 6: 51-55.

3. Miller RW, Rubinstein JH. Tumors in Rubinstein-Taybi syndrome. Am J Med Genet 1995; 56: 112-115.

4. Rubinstein $\mathrm{JH}$, Taybi $\mathrm{H}$. Broad thumbs and toes and facial abnormalities. A possible mental retardation syndrome. Am J Dis Child 1963; 105: 588-608.

5. Roelfsema JH, White SJ, Ariyürek Y, et al. Genetic heterogeneity in Rubinstein- Taybi syndrome: mutations in both the CBP and EP300 genes cause disease. Am J Hum Genet 2005; 76: 572-580.

6. Negri G, Milani D, Colapietro P, et al. Clinical and molecular characterization of Rubinstein-Taybi syndrome patients carrying distinct novel mutations of the EP300 gene. Clin Genet 2015; 87: 148-154.

7. Petrij F, Giles RH, Dauwerse HG, et al. Rubinstein-Taybi syndrome caused by mutations in the transcriptional co-activator CBP. Nature 1995; 376: 348-351.

8. Chrivia JC, Kwok RP, Lamb N, Hagiwara M, Montminy MR, Goodman RH. Phosphorylated CREB binds specifically to the nuclear protein CBP. Nature 1993; 365: 855-859.

9. Lopez-Atalaya JP, Valor LM, Barco A. Epigenetic factors in intellectual disability: the Rubinstein-Taybi syndrome as a paradigm of neurodevelopmental disorder with epigenetic origin. Prog Mol Biol Transl Sci 2014; 128:139-176.

10. Negri G and Gervasini C. 2014. EP300 (E1A binding protein p300) Atlas Genet Cytogenet Oncol Haematol. Available at: http://atlasgeneticsoncology. org//Genes/ P300ID97.html (Accessed February 2, 2017).

11. The Human Gene Mutation Database (HGMD). Available at: http://xx.hgmd.cf.ac.uk/ac/all.php (Accessed February 2, 2017).

12. Bartholdi D, Roelfsema JH, Papadia F, et al. Genetic heterogeneity in Rubinstein-Taybi syndrome: delineation of the phenotype of the first patients carrying mutations in EP300. J Med Genet 2007; 44: 327-333. 